\title{
Calibration of a rockfall simulator with a fragmentation model in a real-scale test
}

\author{
G Matas Universitat Politecnica de Catalunya, Spain \\ N Lantada Universitat Politecnica de Catalunya, Spain \\ J Corominas Universitat Politecnica de Catalunya, Spain \\ R Ruiz-Carulla Universitat Politecnica de Catalunya, Spain \\ A Prades Universitat Politecnica de Catalunya, Spain \\ J Gili Universitat Politecnica de Catalunya, Spain
}

\begin{abstract}
In this contribution, we first present the tool RockGIS, which is a stochastic program for rockfall propagation simulation that takes fragmentation into account considering fractal laws. The results of real-scale field tests and several natural rockfall events inventoried have led to a formulation of a new fragmentation model which has been implemented into RockGIS code. The parameters of the power law that define the sizes of the generated fragments are computed at each impact according to the kinematic conditions. To calibrate the model, we used data from a real-scale rockfall test performed in a quarry. The sizes of the tested blocks were measured manually. Both the size and spatial distribution of the fragments after each release were measured from the orthophotos taken with systematic drone flights. Both rockfall block size distribution (RBSD) and runout distribution obtained after the calibration process matched satisfactory field data, and the spatial distribution of the modelled fragments is qualitatively similar to the one obtained in the test. Finally, the comparison of scenarios with and without fragmentation is presented.
\end{abstract}

Keywords: rockfall simulator, fragmentation, calibration, quarry, hazard

\section{Introduction}

Rockfalls are frequent instability processes in road cuts, open pit mines and quarries, steep slopes, and cliffs. When a rockfall occurs, the initially detached rock mass commonly fragments into smaller blocks during its propagation downslope. Consideration of this fragmentation is relevant for the assessment of hazard since it affects the number of generated blocks, their trajectories and impact energies (Corominas et al. 2019). Most of the rockfall propagation simulators do not consider fragmentation, and in scenarios where it plays a significant role it may lead to an inadequate representation of the phenomena or an inaccurate assessment of risk.

Some authors have developed models based on fractal theory (Ruiz-Carulla et al. 2017; Ruiz-Carulla \& Corominas 2019) that can explain how an initial distribution of blocks (in situ block size distribution, IBSD) changes during propagation due to breakage generating a different distribution (rockfall block size distribution, RBSD). These models have been implemented into RockGIS rockfall simulation code. The implementation of fragmentation requires the consideration of several parameters which increases the difficulty of the calibration process and requires high quality data.

To analyse fragmentation, kinematic features, and the spatial distribution of the fragments, a real-scale experimental study has been carried out in a quarry. The results of the tests have been modelled with the RockGIS code. As calibration criteria, both the runout and volume distributions of fragments were considered. This contribution aims at demonstrating the calibration process of a rockfall fragmentation model. 


\section{Methodology}

This communication shows the calibration of our rockfall simulator (RockGIS) considering fragmentation. The calibration is based on the stopping points and fragment size distributions obtained from a real-scale fragmentation test. To this aim, we first collect the data in fragmentation tests (Gili et al. 2016). The RockGIS simulator was used to reproduce the data observed. The fragmentation model parameters are calibrated in order to fit the simulation results to the real data.

\subsection{RockGIS model}

RockGIS is a GIS-based rockfall propagation model that simulates fragmentation phenomenon during rockfall propagation based on a lumped mass approach (Matas et al. 2017). It is able to simulate both the disaggregation of the blocks forming the initially detached rock mass and the breakage of a single block during its propagation downslope. For disaggregation, no energy loss is assumed. The propagation of the blocks is explicitly computed by integrating movement equations which leads to ballistic trajectories. The impact of a block with the surface is detected using an iterative algorithm and the bisection method into the kinematic integration scheme. The kinematic treatment of the rebound is implemented as described in Gischig et al. (2015). For restitution factors, equations proposed in Wyllie (2014) are used. Collisions between different blocks are not considered along the path, but basic interaction with mitigation structures like fences or walls and buildings is included in a simplified manner (Corominas et al. 2019). The model incorporates a fragmentation module that controls the possible generation of new fragments upon each impact and their trajectories, described in the next section. As a main input data, the simulator requires a digital surface model of the study site, a soil coverage map to allow the use of different parameters depending on the coverage and the position, and volumes of the initial detached blocks.

Each simulation produces a file containing trajectories of the blocks and all the fragments generated including its kinetic energy and the final deposition of the blocks. A specific Python library manages the output information and computes the RBSD produced by the simulation and the final deposition of the fragments.

\subsection{Fractal fragmentation model}

RockGIS considers both the disaggregation of initial rock mass and breakage during propagation. Disaggregation is considered by simulating individually each of the blocks forming the in situ block size distribution. A specific module is triggered every time a block hits the ground to check for breakage during propagation. This module decides if the block remains intact or breaks. In the case of breakage, the module generates the new fragment size distribution based on fractal theory (Ruiz-Carulla et al. 2017; Ruiz-Carulla \& Corominas 2019). The simulator defines, with a certain degree of stochasticity, the trajectories of the created fragments after breakage as new blocks in the simulation.

The fragments' size distribution after breakage is generated with Equation 1. The fragment generation process ends when one of these two conditions are satisfied: 1 ) the last generated fragment is smaller than a user defined minimum volume to avoid a mathematically infinite loop reaching senseless small fragment sizes or 2) the sum of all generated fragments reaches the initial block volume. In the second case, the last fragment is considered to be the difference between the sum of all fragments and the initial block volume.

$$
V_{n}=V_{0} \times L_{\max } \times n^{\frac{-1}{D_{f}}}
$$

where:

$$
\begin{aligned}
& V_{n}=\text { the volume of each fragment generated. } \\
& V_{0}=\text { the initial block volume. } \\
& n \quad=\text { the number of fragments that runs from } 1 \text { to infinite. } \\
& L_{\text {max }}=\text { defines the maximum size fragment generated. } \\
& D_{f}=\text { the fractal dimension that controls the shape of the fragment distribution. }
\end{aligned}
$$


Both $L_{\max }$ and the $D_{f}$ depend on the model parameters $b$ and $q$ as defined in Equations 2 and 3.

$$
\begin{gathered}
L_{\max }=q \times b^{n} \\
D_{f}=3+\frac{\log (1-q)}{\log (b)}
\end{gathered}
$$

where:

$b=$ the geometric factor that controls the fractal proportion between the fragment sizes generated and the initial volume.

$q=$ the probability of survival, controlling if breakage occurs or not, and the degree of breakage considered as a proportion of the blocks that break creating new fragments.

These two model parameters, $b$ and $q$, are related with the normal impact energy with power laws. Each power law is defined by two parameters (b1, b2 and q1, q2). Finally, the calibration is based in these last parameters (b1, b2, q1 and q2) in order to fit the simulation results to the real data obtained in the quarry tests.

\section{Data}

Real-scale rockfall tests were performed in a limestone quarry following the Gili et al. (2016) methodology. A total of 21 blocks ranging from 0.5 to $2.25 \mathrm{~m}^{3}$ were tested in a selected profile. The height of the slope was $19 \mathrm{~m}$ and had an average angle of $74^{\circ}$. At the first impacting point, after an 8-10 $\mathrm{m}$ height fall, the slope was $41^{\circ}$ (Figure 1). The deposited fragments at the base of the slope were cleared after each release.

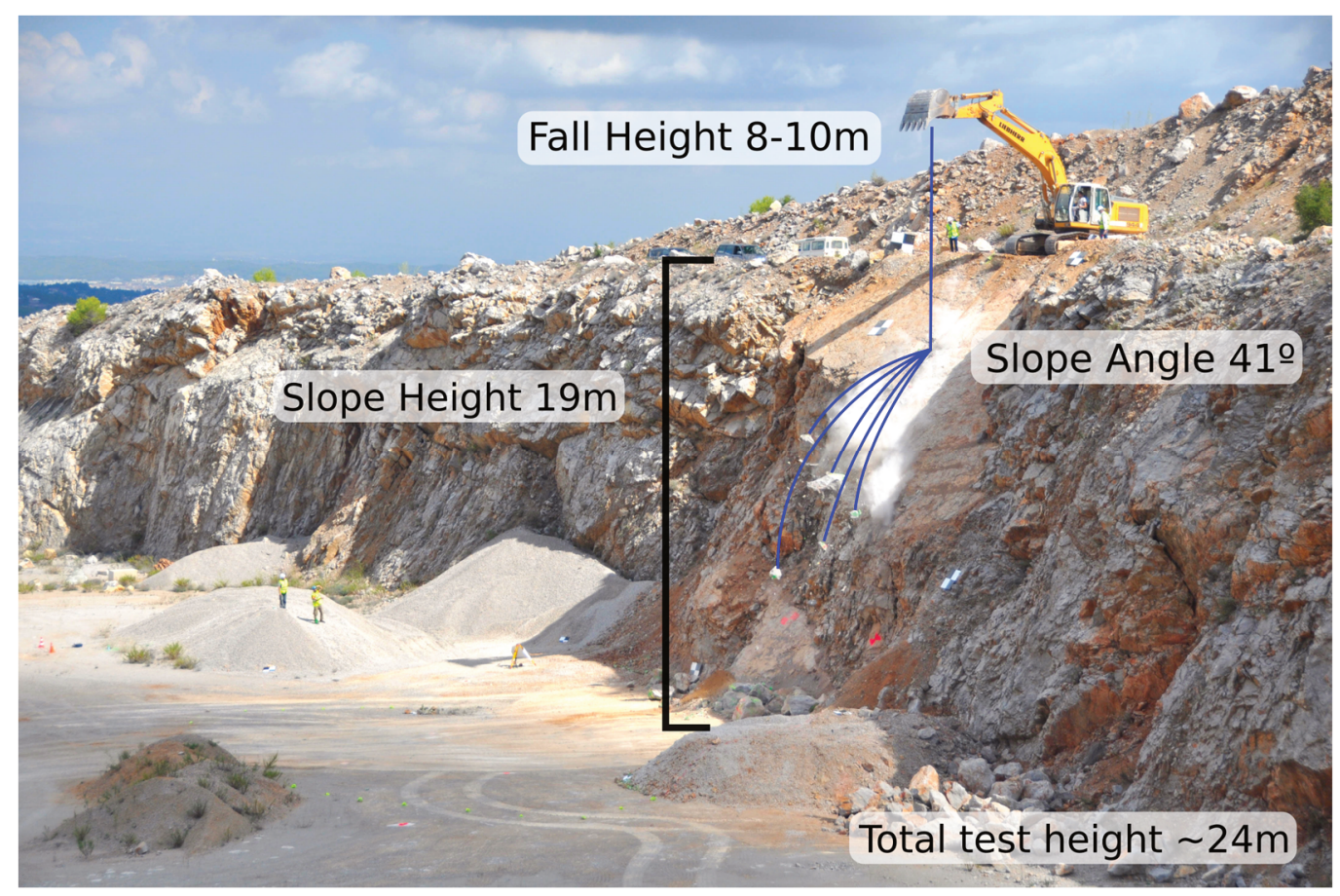

Figure 1 Photograph of the full slope upon which the tests were performed

All initial blocks where manually measured and a 3D model was obtained using terrestrial photogrammetry. Each of these blocks was released by a backhoe, and during its fall three high speed video cameras recorded the trajectories of all generated fragments (Figure 2). 

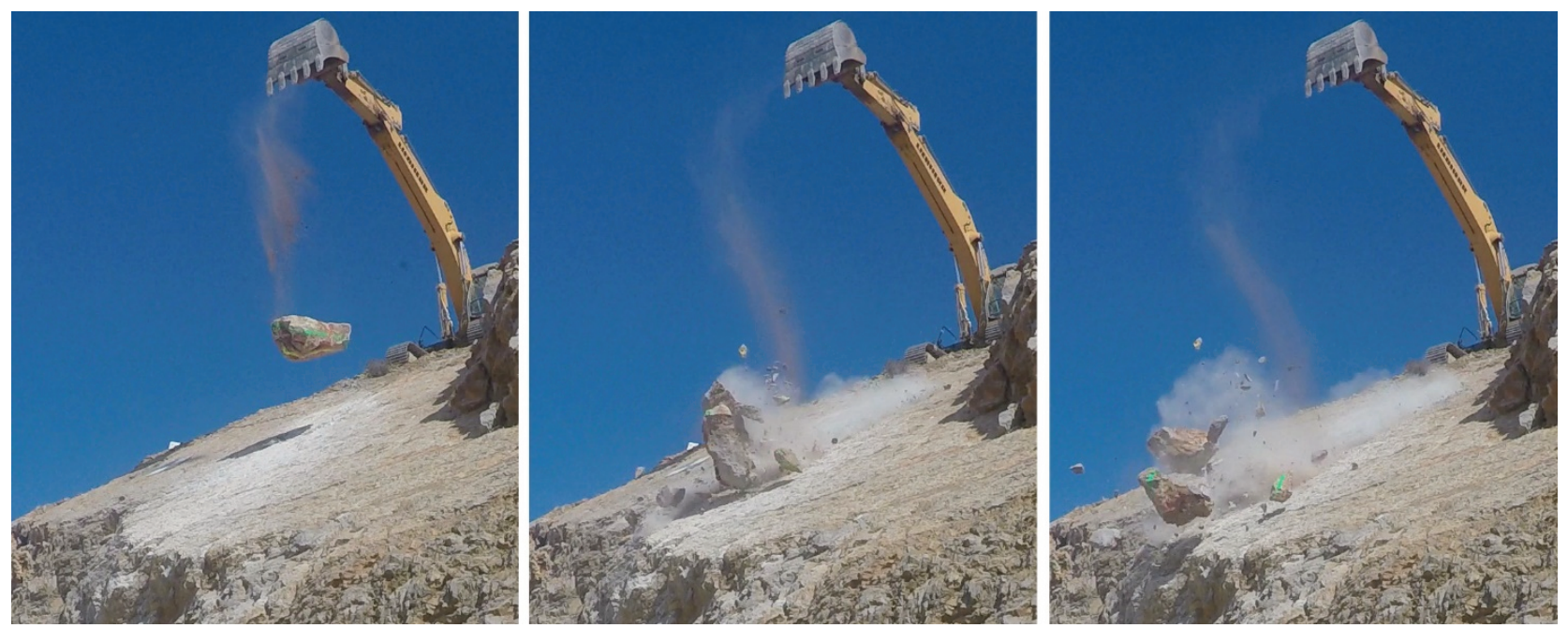

Figure 2 Sequence of a test (block number 15)

After each release, the safety of the scene was checked and then the deposited fragments were manually measured. A drone flight was also performed after each release to generate a 3D model of each block deposit. The orthophoto obtained from the drone flight allowed a precise identification of the deposition point of all fragments and initial impact points (Figure 3 ) and the generation of a surface model of the slope for the simulations. Finally, fragments were removed.

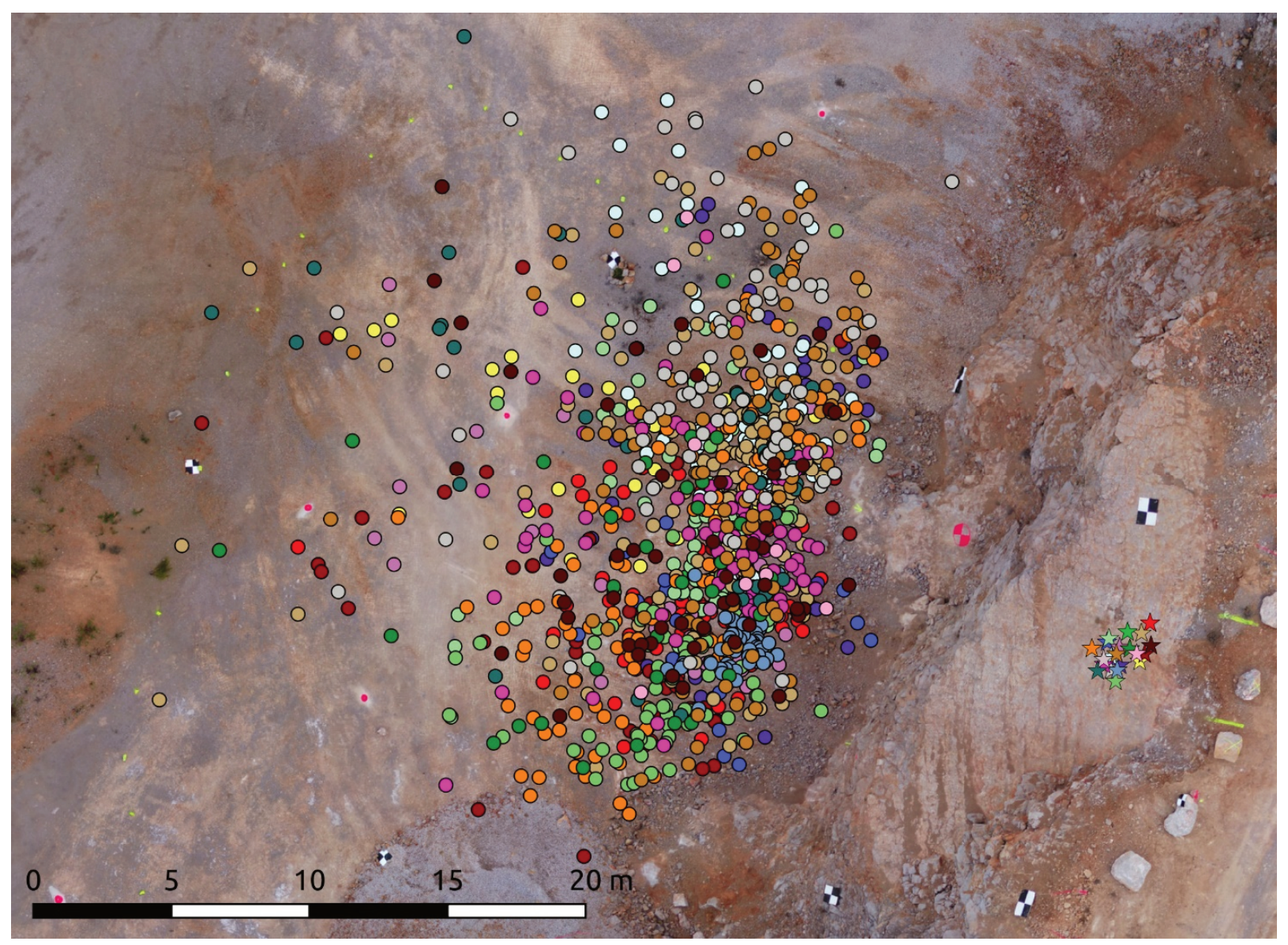

Figure 3 Data from real-scale rockfall tests showing stopping points of all fragments (dots) and first impacts against the slope (stars on the right side) classified by initial release block number by colour 


\section{Calibration}

The calibration process requires setting criteria to compare the simulated and observed data. Two main calibration criteria are considered in this work: 1) the volume distribution and 2) the runout distribution of all generated fragments after the propagation. For statistical representativity, we aggregate the results of all 21 tested blocks. To mathematically compare field and simulation distributions, the statistic $\mathrm{Chi}^{2}$ is used. The calibration process consists of testing multiple sets of different parameter combinations to minimise the value of the $\mathrm{Chi}^{2}$ statistic of both distributions at once. To do so, a Python program was written to first generate the parameter sets within a specified value range. Then each of these sets are used to launch one simulation in RockGIS. For each simulation, both $\mathrm{Chi}^{2}$ results (for the runout and volume distributions) are computed and stored. After all parameter sets were tested, the code selected the lower $\mathrm{Chi}^{2}$ pairs from the list. This process is executed with a parallelisation scheme into a high processing computer.

\section{$5 \quad$ Results}

Both RBSD and runout distribution obtained after the calibration process match satisfactory field data (Figure 4). The spreading of the modelled fragments is qualitatively similar to the one obtained in the test (Figure 5).

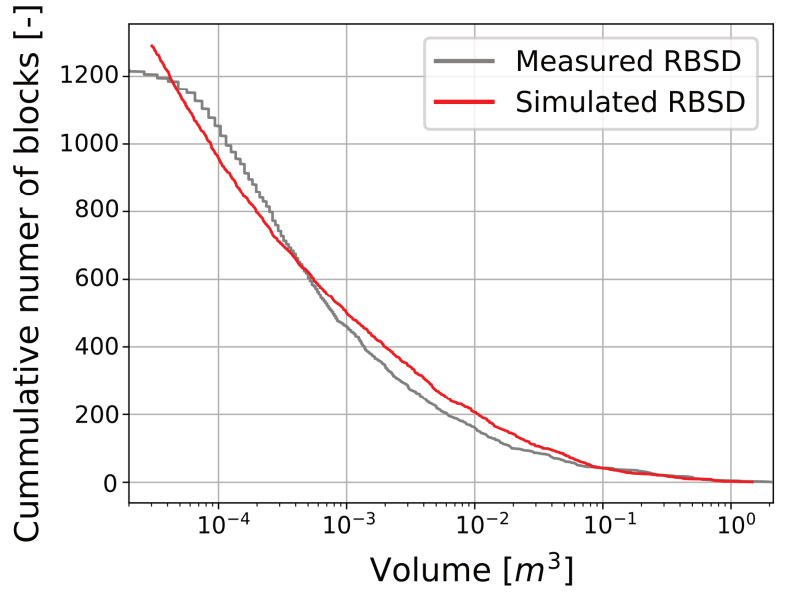

(a)

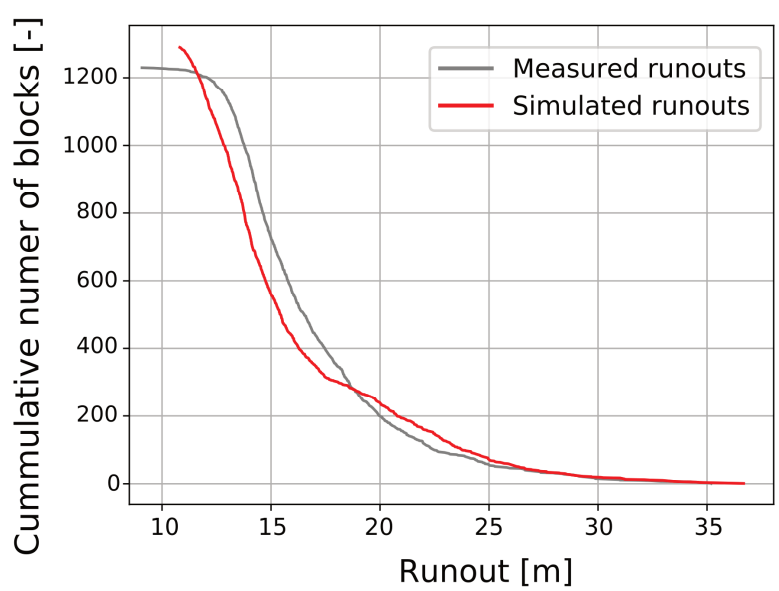

(b)

Figure 4 Comparison between field measurements and simulations results. (a) Rockfall block size distribution; (b) Runout distribution

A total of 1,290 fragments are generated in the simulation while 1,230 were measured in the field. The maximum modelled runout distance obtained is $36.6 \mathrm{~m}$ while the measured was $35.2 \mathrm{~m}$. The largest fragment produced was $2.03 \mathrm{~m}^{3}$ while the largest measured was $1.46 \mathrm{~m}^{3}$.

To illustrate the effect of fragmentation, a simulation was performed deactivating the breakage module. The results show that the runout in the specific conditions of the test increases with fragmentation (Figure 6). This can be explained by the ejection of fragments during breakage, which makes them follow higher parabolic trajectories and travel further. Moreover, after the first impact and breakage at the base of the slope, subsequent impacts of the generated fragments are more tangential to the terrain and dissipate less energy. 


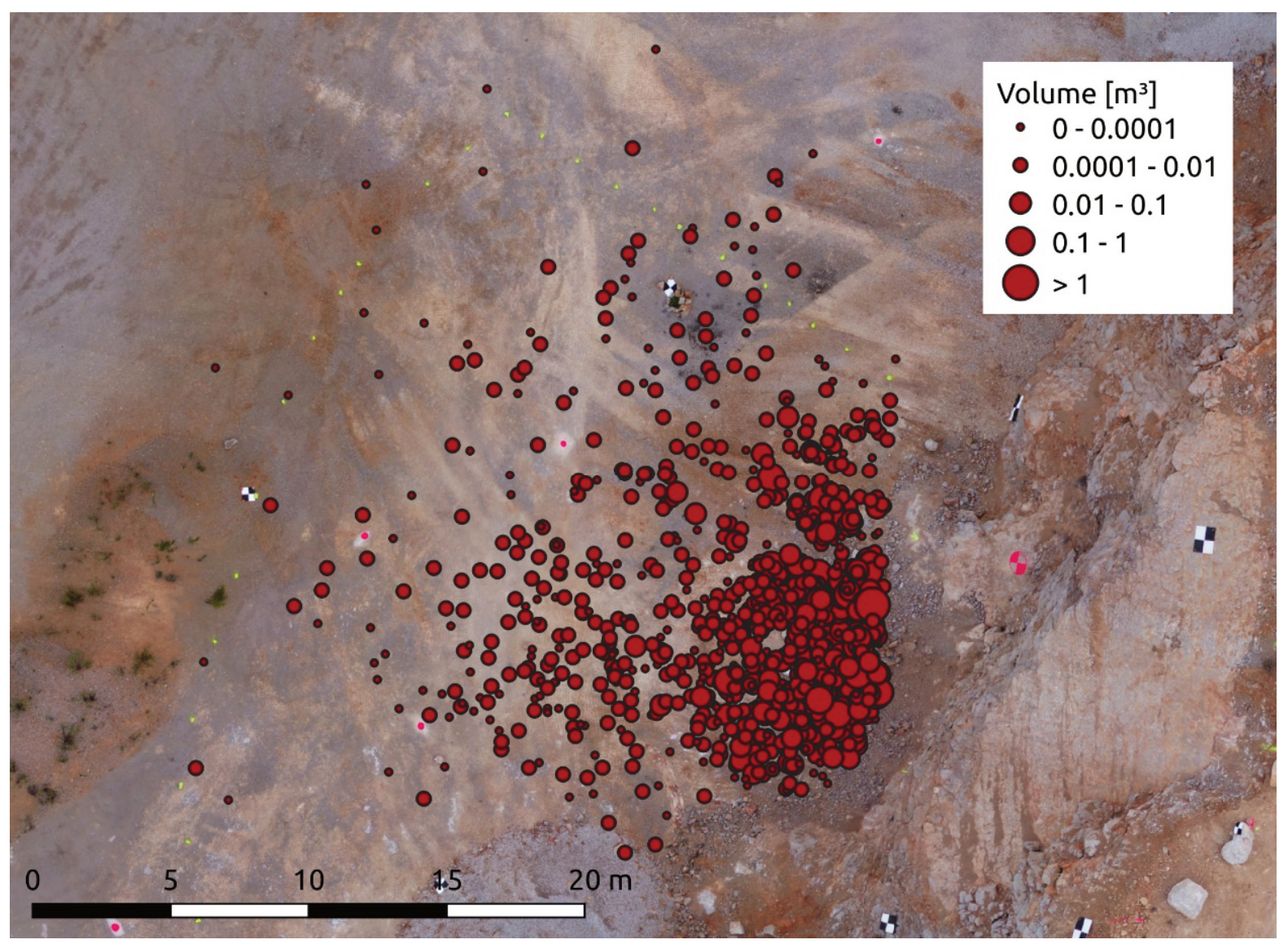

Figure 5 Simulation results: spreading of the fragments along the base of the slope. The size of the dots is proportional to the volume of the fragment

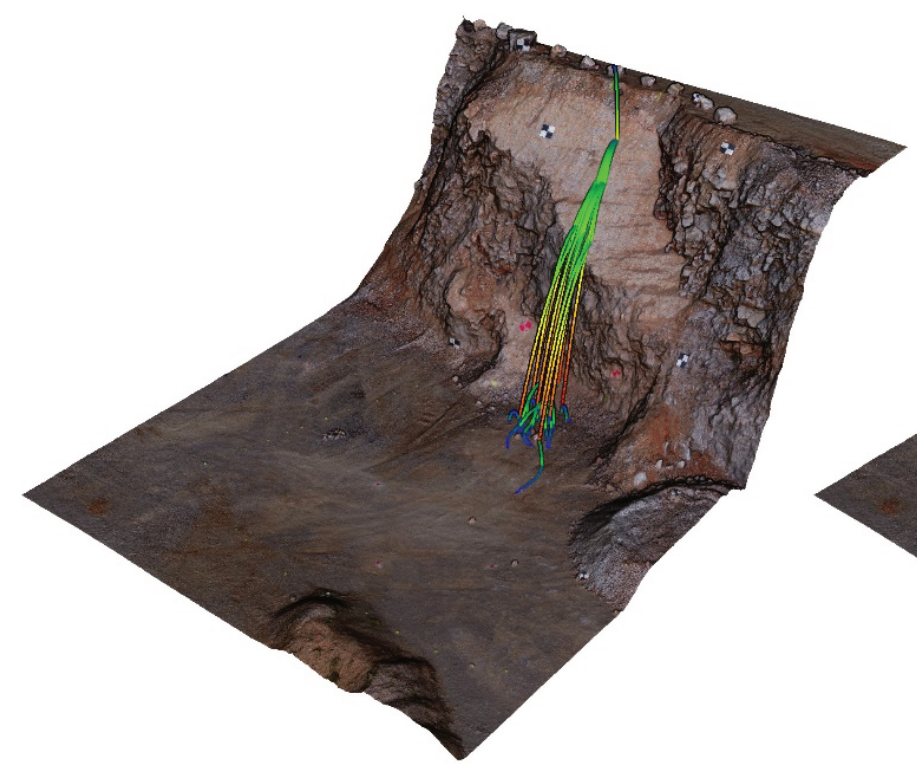

(a)

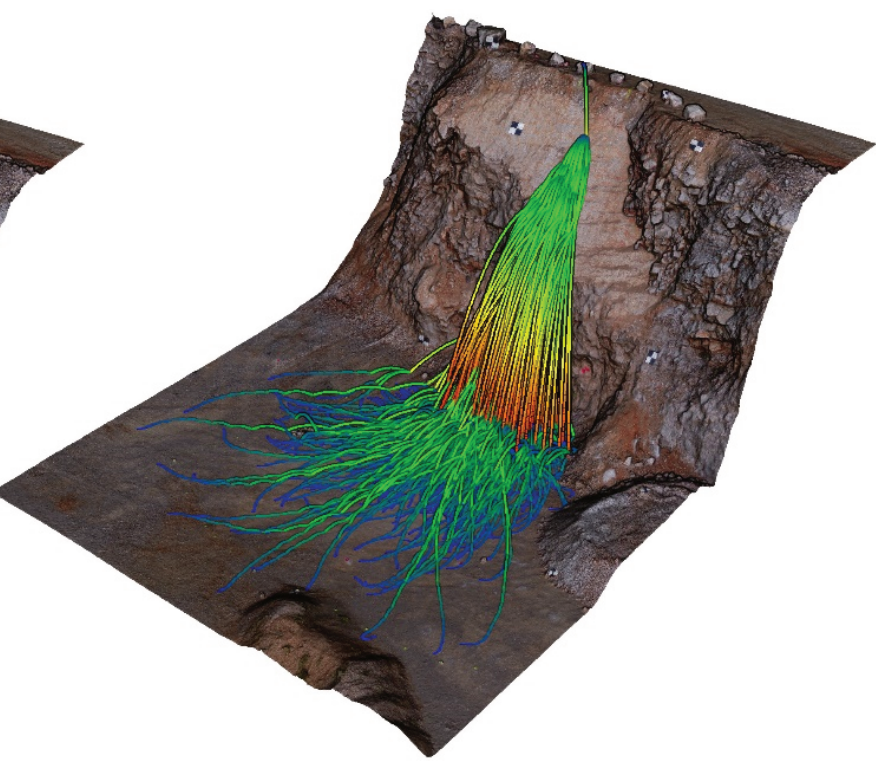

(b)

Figure 6 3D trajectories of all blocks simulated without considering fragmentation (a) and considering fragmentation (b). Note that in a scenario with multiple benches, the number of blocks reaching the second and subsequent benches would increase with fragmentation as seen in (b) 


\section{Conclusion}

The fragmentation process of the blocks during real-scale rockfall tests performed in a quarry has been simulated with the RockGIS code. The results of the runout distribution of the fragments and the volume distribution differ less than $5 \%$ from the observations, and are considered highly satisfactory. The runout distance in some scenarios, such as with abrupt topographical change like in the testing quarry, may be underestimated when not considering fragmentation. Finally, consideration of fragmentation increases the complexity of the process since several more parameters are added to control the breakage laws.

\section{Acknowledgement}

This work has been carried out with the support of the fellowship to the first author and within the framework of the research project Rockmodels financed by the Spanish Ministry of Economy and Competitiveness and the European Regional Development's funds (FEDER), (BIA2016- 75668-P, AEI/FEDER, UE) and by the grants to the first (BES-2014-069795). Finally, the collaboration of Canteras Hermanos Foj (Barcelona metropolitan area, Spain) is gratefully acknowledged.

\section{References}

Corominas, J, Matas, G \& Ruiz-Carulla, R 2019, 'Quantitative analysis of risk from fragmental rockfalls', Landslides, vol. 16, no. 1, pp. 5-21.

Gili, JA, Ruiz-Carulla, R, Matas, G, Corominas, J, Lantada, N, Núñez, MA, Mavrouli, O, Buill, F, Moya, J, Prades, A \& Moreno, S 2016, 'Experimental study on rockfall fragmentation: in situ test design and firsts results', in S Aversa, L Cascini, L Picarelli, C Scavia (eds), Proceedings of the 12th International Symposium on Landslides, CRC Press, Boca Raton, pp. 983-990.

Gischig, V, Hungr, O, Mitchell, A \& Bourrier, F 2015 'Pierre3D: a 3D stochastic rockfall simulator based on random ground roughness and hyperbolic restitution factors', Canadian Geotechnical Journal, vol. 52, pp. 1-14.

Matas, G, Lantada, N, Corominas, J, Gili, JA, Ruiz-Carulla, R \& Prades, A 2017, 'RockGIS: A GIS-based model for the analysis of fragmentation in rockfalls', Landslides, vol. 14, no. 5, pp. 1565-1578, https://dx.doi.org/10.1007/s10346-017-0818-7

Ruiz-Carulla, R, Corominas, J \& Mavrouli, O 2017, 'A fractal fragmentation model for rockfall', Landslides, vol. 14, no. 3, pp. 875-889, https://dx.doi.org/10.1007/s10346-016-0773-8

Ruiz-Carulla, R \& Corominas, J 2019, 'Analysis of rockfalls by means of a fractal fragmentation model', Journal of Rock Mechanics and Rock Engineering, https://dx.doi.org/10.1007/s00603-019-01987-2

Wyllie, DC 2014, Rock Fall Engineering: Development and Calibration of an Improved Model for Analysis of Rock Fall Hazards on Highways and Railways, PhD thesis, The University of British Columbia, Vancouver. 
